\title{
TEMPERATURA DE LA SUPERFICIE TERRESTRE EN DIFERENTES TIPOS DE COBERTURA DE LA REGIÓN ANDINA COLOMBIANA
}

\section{Land surface temperature in different land covers of the Andean Region of Colombia}

\author{
Andrés Felipe Carvajal \\ Universidad Nacional de Colombia, Bogotá, Colombia. \\ afcarvajalv@unal.edu.co \\ José Daniel Pabón \\ Universidad Nacional de Colombia, Bogotá, Colombia. \\ jdpabonc@unal.edu.co
}

Artigo recebido em 27/05/2013 e aceito para publicação em 16/01/2014

RESUMEN: Se evaluó la relación de los índices de vegetación de diferencia normalizada (IVDN) y de agua de diferencia normalizada (IADN) con la temperatura de la superficie terrestre (TST), por medio de la utilización de imágenes Landsat de la cuenca del río La Vieja, en la región Andina colombiana. Se evaluaron las coberturas de selva Andina, plantación forestal, café, pasto y zona urbana. Se identificaron correlaciones negativas entre los índices y la TST, y se encontraron diferencias significativas $(\mathrm{p}<0.01)$ entre tipos de cobertura. El pasto presentó TST promedio mayor a la zona urbana, y el café menor a la de bosques y plantaciones. Las transiciones de pastos hacia plantación, selva Andina y café mostraron disminuciones en TST hasta de $9^{\circ} \mathrm{C}$, de zona urbana a café hasta de $8{ }^{\circ} \mathrm{C}$, mientras que de zona urbana a pastos se incrementó hasta en $1.28^{\circ} \mathrm{C}$. Se concluyó que los pastos pueden tener mayor capacidad para favorecer el calentamiento de la superficie que la zona urbana y, que el café se asemeja a los bosques y plantaciones en la capacidad de enfriamiento. Se planteó que no solo se debe prestar atención a las islas de calor urbanas, porque en las zonas rurales también existen islas de calor asociadas con actividades pecuarias, las cuales no han sido estudiadas con profundidad.

Palabras-clave: Índice de Vegetación de Diferencia Normalizada (IVDN), Índice de Agua de Diferencia Normalizada (IADN), Sensores Remotos, Isla de Calor, Landsat.

ABSTRACT This study assessed the relationship of Normalized Difference Vegetation Index (NDVI) and Normalized Difference Water Index (NDWI) with land surface temperature (LST), using Landsat images of La Vieja river watershed, in the Andean region of Colombia. We evaluated land covered by Andean forest, forest plantation, coffee, pasture and urban area. Negative correlations between indices and LST were identified, and significant differences $(\mathrm{p}<0.01)$ between land cover types were found. Pasture presented mean LST greater than urban area, and coffee lower than forests and plantations. Transitions from pasture to Andean forest, plantation and coffee showed decreases in LST up to $9^{\circ} \mathrm{C}$, from urban area to coffee up to $8^{\circ} \mathrm{C}$, while from urban area to pasture increased up to $1.28^{\circ} \mathrm{C}$. We concluded that pasture can have higher capacity than urban area to promote heating of land surface, and that coffee is similar to forests and plantations in its cooling capacity. It was suggested that there is to pay attention not only to the urban heat islands but to the rural heat islands associated to livestock activities that have not been deeply studied.

Keywords: Normalized Difference Vegetation Index (NDVI), Normalized Difference Water Index (NDWI), Remote Sensing, Heat Island, Landsat. 


\section{INTRODUCCIÓN}

Las transformaciones en la cobertura de la superficie terrestre (por actividades como la deforestación, la urbanización y las prácticas agropecuarias, principalmente) hacen parte de los procesos de cambio global y, pueden repercutir de manera negativa sobre las características biofísicas de la superficie y su interacción con la atmósfera. La sustitución de la vegetación puede afectar aspectos del balance de radiación como el albedo y la absorción de radiación solar, así como el intercambio de calor y humedad entre la superficie y la atmósfera (Stohlgren et al., 1998). Algunas investigaciones han demostrado que la partición de calor sensible y calor latente, y por lo tanto la respuesta de la temperatura de la superficie, es modulada por la cobertura vegetal y el contenido de agua sobre el suelo (OWEN et al., 1998). Por lo tanto, el estudio de la distribución espacial de las coberturas vegetales en paisajes altamente transformados, y su relación con la TST, cobra importancia porque se convierte en una manera de identificar la contribución de los procesos de transformación de la superficie con la problemática del calentamiento global.

Diferentes trabajos se han enfocado en el análisis de la TST de las ciudades para compararla con la de zonas rurales circundantes e identificar islas de calor urbanas (XIAN; CRANE, 2006; ZHANG et al., 2009; YUAN; BAUER, 2007; STATHOPOULOU; CARTALIS, 2007; NICHOL et al., 2009; MALLICK et al., 2008). Otros estudios han abarcado áreas más extensas (JULIEN et al., 2006; NETELER, 2010; Li et al., 2004), pero no han hecho suficiente énfasis en los impactos que generan las transformaciones de las coberturas en las zonas rurales. La preocupación surge porque las ciudades son una cobertura concentrada espacialmente, mientras que las coberturas vegetales tienen una distribución geográfica más amplia y deben ser evaluadas a profundidad, para identificar la forma en que su distribución espacial coincide con las variaciones en la temperatura de la superficie y pueden contribuir con el calentamiento del planeta.

En épocas recientes, el uso de la teledetección ha contribuido con la obtención de información del estado de las coberturas y ha permitido el estudio de su relación con la temperatura radiante. Incluso, se afirma que los sensores remotos termales infrarrojos son la única posibilidad que existe para obtener datos de temperatura sobre áreas extensas de la superficie terrestre, a diferentes resoluciones espaciales y temporales (SOBRINO et al., 1994; COLL et al., 2005). Por lo tanto, el conocimiento de la TST en escalas espaciales y temporales más amplias, se convierte en una necesidad para diversas aplicaciones, entre las que se encuentran los estudios climáticos y ambientales. Xian y Crane (2006) plantean que la TST es un indicador de cambio ambiental asociado con las actividades humanas, razón por la cual se convierte en un factor importante en los estudios de cambio global, específicamente en lo que respecta al balance de radiación y el control del calentamiento global (SRIVASTAVA et al., 2009).

La región Andina colombiana ha presentado una presión antropogénica alta, debido a que gran parte de la población del país se ha concentrado en ella y ha utilizado las tierras en forma intensiva (MARÍN et al., 2008). Específicamente la cuenca del río La Vieja, a pesar de ser una zona con un área importante sembrada en café, ya presentaba en el año 2002 un $34.8 \%$ de su área total cubierta por pastos; lo que según CRQ et al. (2005) se pudo presentar en parte por la crisis en el sector cafetero. La disminución en los precios internacionales de este producto agrícola después del año 1989, generó una crisis económica que pudo haber promovido la sustitución de cultivos de café por sistemas ganaderos, aunque dicha sustitución en la cuenca también pudo estar relacionada con la tendencia que han presentado los procesos de colonización de tierras, en los que inicialmente se ha comenzado con prácticas agrícolas y finalmente se han introducido pastizales para ganado bovino (ETTER et al., 2005), como una forma de mantener esas tierras que han sido sobre utilizadas y que ya no son altamente productivas. A pesar de esto, la cuenca todavía conserva áreas importantes con vegetación natural, las cuales se localizan en las zonas donde la altura sobre el nivel del mar aumenta y la topografía es más escarpada, coincidiendo con lo planteado por Wilson et al. (2005), quienes afirman que los relictos de vegetación natural en paisajes fuertemente transformados, por lo regular se encuentran en tierras económicamente marginales caracterizadas por suelos menos fértiles con pendientes más pronunciadas y de difícil acceso. 
De lo anterior, se puede deducir que la cuenca del río La Vieja es una región que ha estado sujeta a un proceso fuerte de transformación del paisaje, lo que puede haber generado cambios en la temperatura de la superficie que podrían estar contribuyendo con la problemática del calentamiento global. Por lo tanto, este trabajo intentó identificar las variaciones en la TST asociadas con las principales coberturas presentes en la cuenca, por medio del análisis de la relación entre IVDN, IADN y TST; y con la evaluación de la distribución espacial de la TST en zonas con transiciones entre diferentes tipos de cobertura.

\section{MÉTODOS}

\section{Área de estudio}

La cordillera de los Andes en Colombia se divide en tres sistemas montañosos: la cordillera Occidental, la Central y la Oriental. La cuenca del río La Vieja se encuentra localizada en la vertiente occidental de la cordillera Central, en la zona que se conoce como la región Andina colombiana. Posee una extensión de $2763 \mathrm{~km}^{2}$ y en el año 2002 la mayor cobertura de la cuenca eran los pastizales que representaban el 34,8\% del área total, seguido por cultivos $(27,4 \%)$ principalmente de café, y relictos de selva Andina y plantaciones forestales $(21,4 \%$ ) (CRQ et al., 2005). La cuenca presenta diferencias altitudinales importantes que varían entre 893 y 4799 m, y la zona más alta se localiza en la región occidental (Figura 1), donde aún se mantienen áreas importantes con coberturas naturales como relictos de selva Andina y vegetación de páramo; aunque también es posible encontrar plantaciones forestales y algunas áreas en pastizal que son producto del establecimiento de sistemas ganaderos en tierras altas.

La región central presenta alturas menores, y es el área donde se concentra la mayor actividad agrícola de la cuenca, con predominancia de cultivos de café. Mientras que la región occidental corresponde con las tierras más bajas, con menores precipitaciones y temperaturas más altas. Es la zona en la que se ha transformado gran parte de las coberturas hacia pastos y se pueden apreciar algunos relictos de selva Andina principalmente en la ribera de los ríos.

\section{Procesamiento de imágenes}

Se utilizaron cuatro imágenes Landsat de diferentes periodos para identificar las coberturas de la tierra presentes en la cuenca (Tabla 1). Se realizó corrección geométrica con puntos de control levantados en campo y cartografía local. Posteriormente se aplicó corrección atmosférica para cada una de las escenas utilizando el método de la sustracción del objeto oscuro (CHAVEZ, 1988) y se procedió a hacer una clasificación supervisada con la ayuda de fotografías aéreas de los años 1989 y 2003. 
Figura 1. Localización del área de estudio.

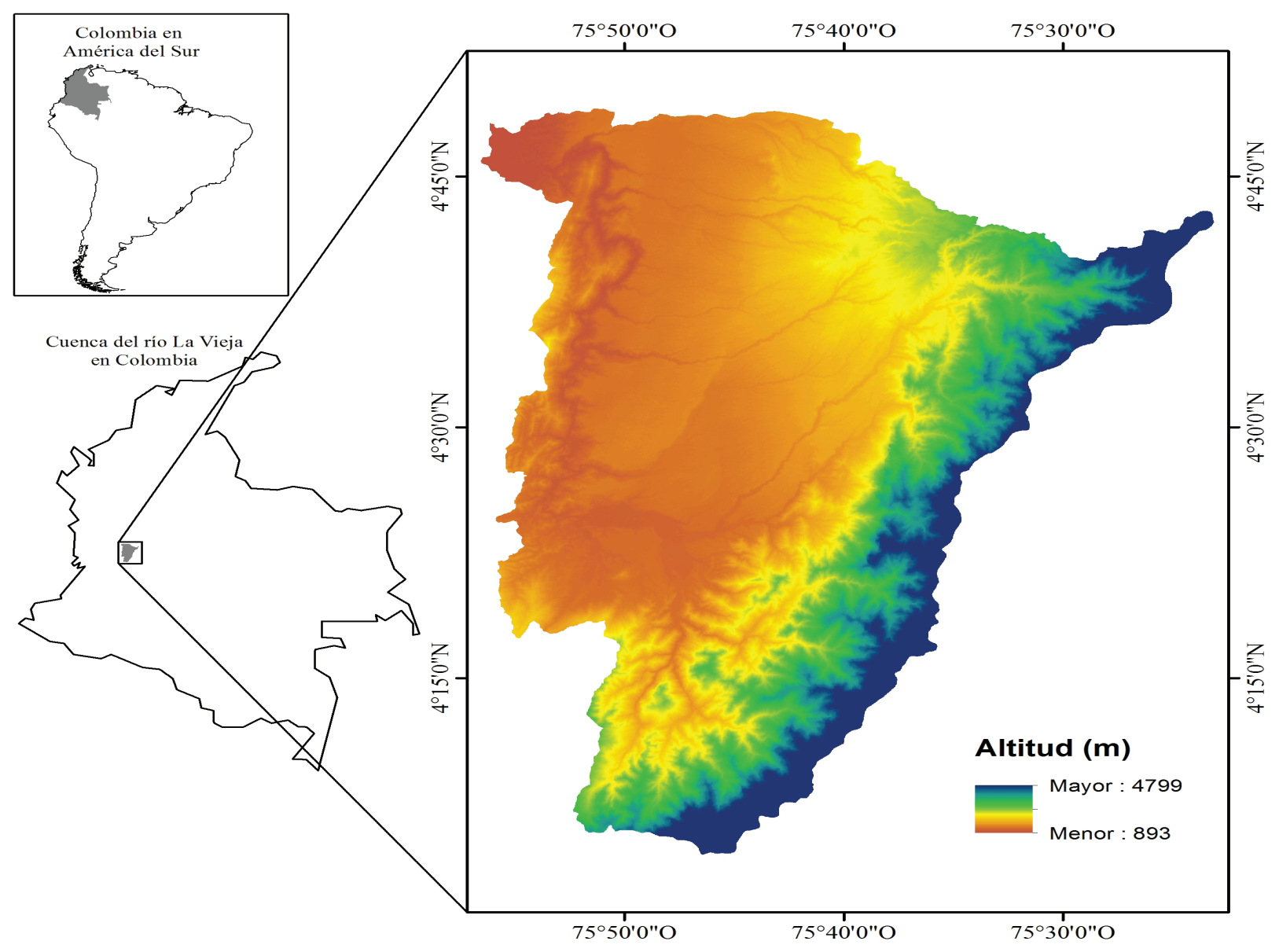

Organización de los autores.

Tabla 1. Características de las imágenes Landsat utilizadas en el estudio.

\begin{tabular}{ccccc}
\hline Fecha & Columna/Fila & Satélite & $\begin{array}{c}\text { Resolución espacial bandas } \\
1-5,7(\mathrm{~m})\end{array}$ & $\begin{array}{c}\text { Resolución espacial banda } \\
6 \text { (térmica) }(\mathrm{m})\end{array}$ \\
\hline $1989 / 08 / 07$ & $09 / 57$ & Landsat-4 & 30 & 120 \\
\hline $1997 / 08 / 21$ & $09 / 57$ & Landsat-5 & 30 & 60 \\
\hline $1998 / 05 / 20$ & $09 / 57$ & Landsat-5 & 30 & 60 \\
\hline $2002 / 07 / 26$ & $09 / 57$ & Landsat-7 & 30 & 60 \\
\hline
\end{tabular}

Organización de los autores. 
Cada una de las imágenes Landsat fue calibrada a valores de radiancia espectral utilizando los valores especificados en los respectivos metadatos y la siguiente ecuación (CHANDER; MARKHAM, 2003): $\mathrm{L}_{1}=\left(\left(\operatorname{LMAX}_{1}-\mathrm{L}\right.\right.$
cal $\left.\left.-\mathrm{Q}_{\text {cal min }}\right)+\mathrm{LMIN}_{1}\right)$

donde; sr $\mu \mathrm{m})$

$\mathrm{L}_{1}=$ radiancia spectral expresada en $\mathrm{W} /\left(\mathrm{m}^{2}\right.$

$\mathrm{Q}_{\text {cal }}=$ valor del Número Digital (ND) del pixel

$\mathrm{Q}_{\text {cal max }}=$ máximo ND de la imagen correspondiente con LMAX $_{1}$ con LMIN

$\mathrm{Q}_{\text {cal min }}=$ mínimo ND imagen correspondiente

$\mathrm{LMAX}_{1}=$ radiancia correspondiente con el ND máximo de la imagen expresada en $\mathrm{W} /\left(\mathrm{m}^{2} \mathrm{sr} \mu \mathrm{m}\right)$

$\mathrm{LMIN}_{1}=$ radiancia correspondiente con el ND mínimo de la imagen expresada en $\mathrm{W} /\left(\mathrm{m}^{2} \mathrm{sr} \mu \mathrm{m}\right)$

Posteriormente los Números Digitales (ND) de los pixeles en la banda seis (banda térmica) fueron convertidos a temperatura de superficie por medio de algoritmos ya establecidos (Chander et al., 2009):

$\mathrm{T}_{\mathrm{s}}=k_{2} / \ln \left(\left(k_{l} / \mathrm{L}_{1}\right)+1\right)$

donde;

$\mathrm{T}_{\mathrm{s}}=$ Temperatura de superficie expresada en grados Kelvin (k) sr $\mu \mathrm{m})$

$\mathrm{L}_{1}=$ radiancia spectral expresada en $\mathrm{W} /\left(\mathrm{m}^{2}\right.$

$K_{I}($ Landsat 4$)=671.72 \mathrm{~W} /\left(\mathrm{m}^{2} \mathrm{sr} \mu \mathrm{m}\right)$

$K_{2}($ Landsat 4$)=1284.3 \mathrm{k}$

$K_{l}($ Landsat 5$)=607.76 \mathrm{~W} /\left(\mathrm{m}^{2} \mathrm{sr} \mu \mathrm{m}\right)$

$K_{2}($ Landsat 5$)=1260.56 \mathrm{k}$

$K_{l}($ Landsat 7$)=666.09 \mathrm{~W} /\left(\mathrm{m}^{2} \mathrm{sr} \mu \mathrm{m}\right)$

$K_{2}($ Landsat 7$)=1282.71 \mathrm{k}$

Después de obtener la temperatura de superficie en grados Kelvin, se llevó a cabo la conversión a grados Celsius.

El procesamiento de las imágenes se realizó con el programa ENVI versión 4.8 (Research Systems, Inc.) y las salidas gráficas se elaboraron en el programa Arcmap versión 10.0 (ESRI, Inc.).

Para identificar las características de cada una de las coberturas se utilizaron los índices de Vegetación de Diferencia Normalizada (IVDN) y de Agua de Diferencia Normalizada (IADN). El IVDN es un índice que separa la vegetación verde de otras coberturas, porque la clorofila de la vegetación absorbe la luz roja para la fotosíntesis y refleja las longitudes de onda del infrarrojo cercano debido a la dispersión causada por la estructura interna de las hojas (WILSON; SADER, 2002). Se calcula por medio de una correlación de reflectancia de la región del rojo $(\mathrm{R})$ y el infrarrojo cercano (IRC) del espectro electromagnético (Rouse et al., 1974). Utilizando las bandas 3 y 4 de las imágenes Landsat se calculó por medio de la siguiente fórmula:

IVDN= IRC (banda 4) $-\mathrm{R}$ (banda 3)/ IRC (banda 4) + R (banda 3)

El IADN es un índice que responde a las moléculas líquidas de agua en el dosel de la vegetación, de manera que diferencia la vegetación más seca de la vegetación más verde, siendo sensible a la capacidad de absorción de humedad de las hojas. Se calcula correlacionando la reflectancia del infrarrojo cercano (IRC) y el infrarrojo medio (IRM) (GAO, 1996). Con las bandas 4 y 5 de las imágenes Landsat se calculó por medio de la siguiente fórmula:

IADN $=$ IRC (banda 4) - IRM (banda 5)/ IRC (banda 4) + IRM (banda 5)

\section{Análisis estadístico}

Las imágenes se seleccionaron tomando como criterio que en cada una de ellas fuera posible seleccionar 2000 pixeles libres de nubes para cada una de las coberturas de interés (selva Andina, plantación forestal, cultivo de café, pasto y zona urbana). De las cuatro escenas Landsat se tomaron 8.000 pixeles para cada una de las cinco coberturas evaluadas, con lo que se obtuvo un número total de 40.000 muestras. Se identificó el efecto de la altitud sobre la temperatura de superficie por medio de análisis de correlación y de regresión lineal entre los valores de TST y la altitud de cada uno de los pixeles en los que se tomaron las muestras. Para ello, se utilizó un modelo digital de elevación SRTM con resolución espacial original de $90 \mathrm{~m}$ y disponible de manera gratuita en: http://srtm. csi.cgiar.org/. Posteriormente se recalculó el tamaño de pixel para obtener una resolución de $30 \mathrm{~m}$, con el fin de hacer compatible el modelo con las imágenes Landsat utilizadas. 
Se encontró una correlación de 0.8 entre las dos variables y una disminución en TST de $0.007^{\circ} \mathrm{C}$ por cada metro de incremento sobre el nivel del mar (Figura 2). Después se calculó la diferencia de altura (DA) entre cada pixel y la altura más baja de la cuenca (893 m) y con la pendiente de la recta obtenida con el modelo de regresión (-0.007) se construyó una fórmula que permitió calcular la temperatura ajustada de la superficie, en la que se eliminó el efecto de la altitud adicionando $0.007^{\circ} \mathrm{C}$ por metro de diferencia. La fórmula es la siguiente;

$$
\mathrm{TST}_{\text {(ajustada) }}=\mathrm{TST}+0.007 * \mathrm{DA}
$$

La TST ajustada se utilizó en los análisis de varianza no paramétrica en los que se compararon las medias de los índices y la temperatura por cobertura. También se utilizó para comparar las transiciones entre coberturas.

Por el contrario, en los análisis de correlación y de regresión lineal IVDN-TST, IADN-TST se utilizó la temperatura sin ajustar. Los análisis estadísticos se realizaron con el pograma Infostat versión 2012 (DI RIENZO et al., 2012).

Figura 2. Regresión lineal entre TST y altitud.

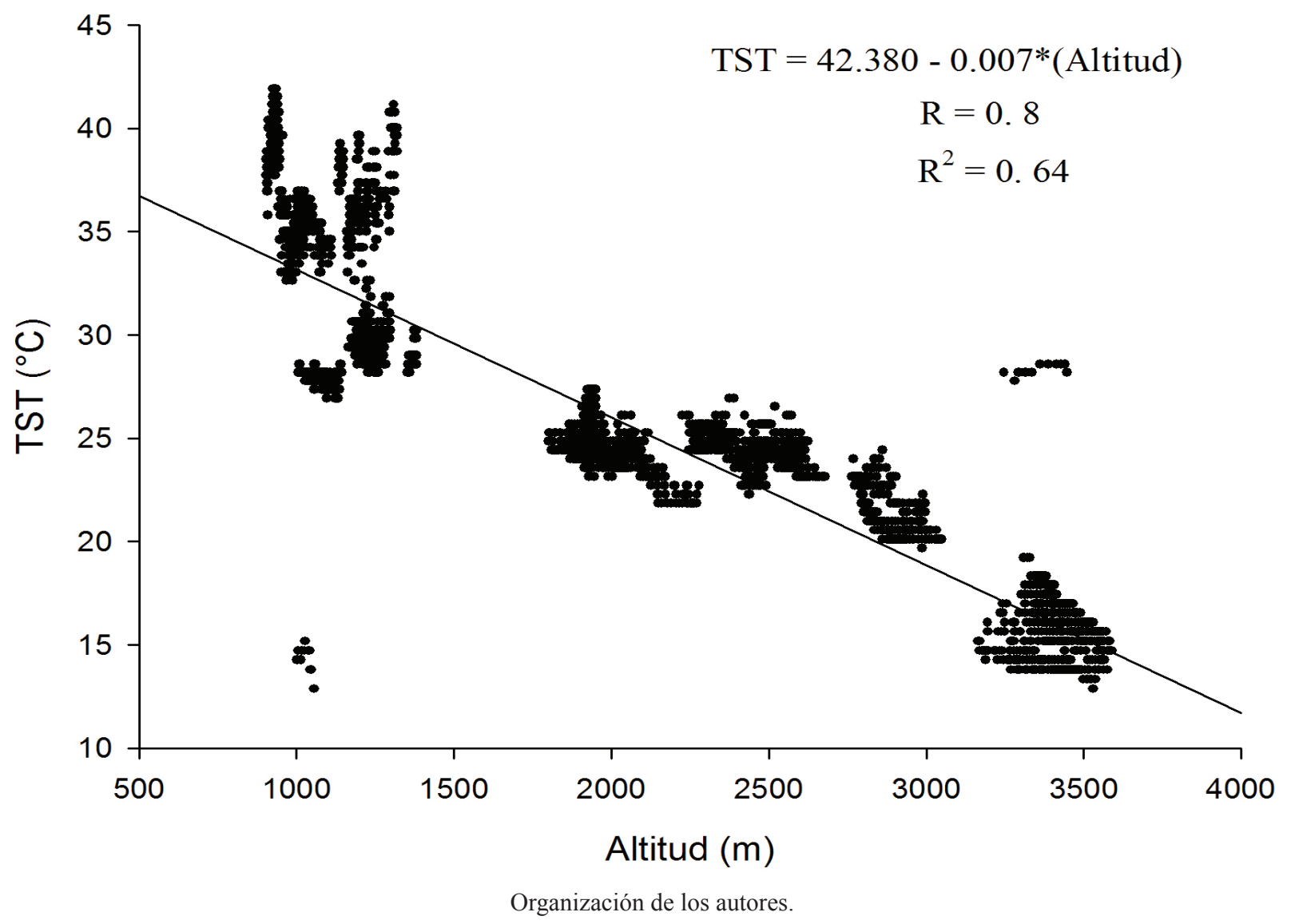




\section{RESULTADOS Y DISCUSIÓN}

\section{IVDN, IADN y TST por tipo de cobertura}

Las coberturas de la superficie terrestre analizadas mostraron un patrón de diferenciación en función de los valores de IVDN y IADN. Los pastos y la zona urbana presentaron características similares entre sí, pero diferentes a los de plantaciones forestales, selva Andina y cultivos de café. La vegetación arbórea de alto (selva Andina y plantación forestal) y bajo porte (café) se caracterizó por presentar IVDN y IADN altos.

En las cuatro imágenes Landsat evaluadas la cobertura urbana se separó de las demás por presentar los valores más bajos para los dos índices, mientras que la separación de los pastos no fue tan evidente en todas las épocas (Figura 3). Esto se pudo dar debido a características fenológicas de la vegetación que responden a eventos climáticos. Las escenas 1997/08/21, 1998/05/20 y 2002/07/26 corresponden con meses de ocurrencia del fenómeno El Niño, lo que indica que se pudo haber disminuido la precipitación y haber generado estrés hídrico en las coberturas de selva Andina, plantación forestal y café, razón por la cual se observan cercanas al patrón de pastos. Por el contrario, la imagen 1989/08/07 muestra la clara separación de las coberturas arbóreas en relación con las gramíneas y la zona urbana, lo que pudo corresponder con altos contenidos de humedad en la vegetación, debido a que la imagen pertenece a un año en el que los primeros meses concuerdan con el fenómeno La Niña, lo que pudo influir en el aumento de las precipitaciones y por lo tanto en las condiciones de la vegetación, incrementando así los valores de IADN.

El IVDN no mostró indicios de variaciones considerables entre las diferentes épocas de análisis, lo que podría indicar que este índice de vegetación es más estable que el IADN en relación con la variabilidad climática. Algunos estudios han encontrado que el IVDN responde con mayor claridad a las variaciones climáticas en regiones áridas y semiáridas, donde la vegetación es escasa y la precipitación es baja (JI; PETERS, 2003; Wang et al., 2003), condiciones que son contrarias a las de la cuenca del río La Vieja, que presenta una alta cobertura vegetal y se localiza en una región con precipitaciones frecuentes. Por su parte, Wang et al. (2011) encontraron que el IADN es más sensible que el IVDN a la variación en la disponibilidad de agua, lo que hace que refleje con mayor facilitad los efectos de las temporadas secas y lluviosas sobre las características de la vegetación.

Es importante resaltar que el cultivo de café, a pesar de poseer una estructura vegetal diferente a la de la selva Andina y las plantaciones forestales, se asemeja en relación con los contenidos de humedad y clorofila en las hojas. 
Figura 3. Relación entre IVDN y IADN en cada una de las imágenes Landsat analizadas.
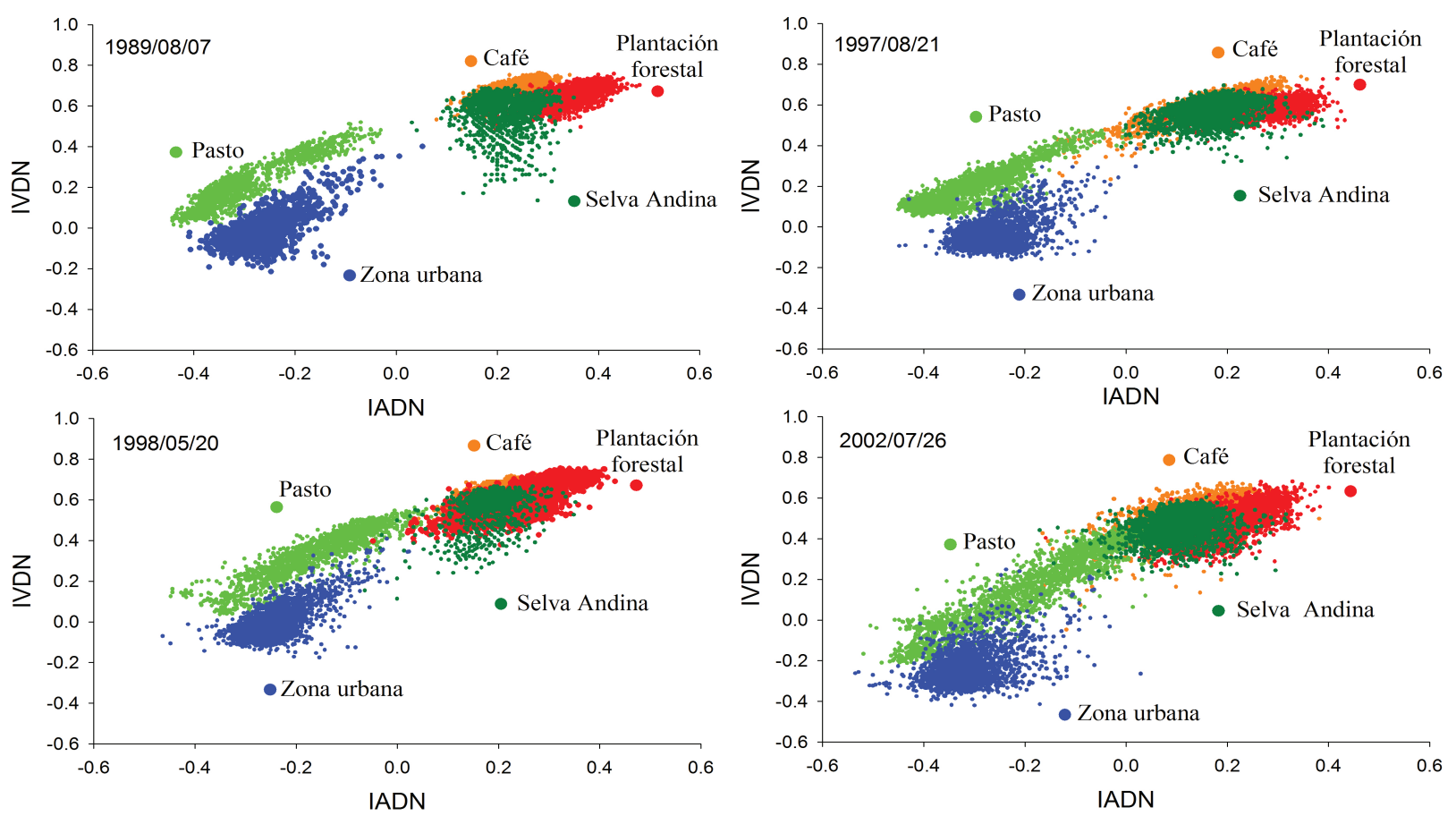

Organización de los autores.

Las medias de los índices para cada una de las coberturas presentaron diferencias estadísticamente significativas $(\mathrm{p}<0.01)$. En la cobertura urbana predominaron valores negativos en el IVDN debido a la ausencia de vegetación. De las coberturas vegetales, los pastos presentaron los valores más bajos en las cuatro escenas, con variaciones entre 0.15 y 0.33 , los de selva Andina estuvieron entre 0.46 y 0.57 , los de plantación forestal entre 0.47 y 0.64 y los de café entre 0.52 y 0.68 (Tabla 2). El cultivo de café se caracterizó por presentar los promedios de IVDN más altos en todas las imágenes, superando coberturas con dosel más amplio como selva y plantaciones forestales.

El IADN fue negativo para la cobertura urbana y los pastos, incluso en las escenas 1989/08/07 y 1998/05/20 los valores de los pastos fueron menores que los de la zona construida. Por el contrario, las plantaciones forestales se caracterizaron por el IADN más alto seguidas por los cultivos de café (Tabla 2).

En cuanto a la TST promedio, se determinó que los pastos pueden llegar a presentar temperaturas superiores a las de la cobertura urbana. Inclusive, en las escenas 1989/08/07 y 1998/05/20, la mayor tem- peratura promedio correspondió con zonas cubiertas por pastos, con valores de 38.64 y $42.27^{\circ} \mathrm{C}$ respectivamente; mientras que en la cobertura urbana las temperaturas fueron de 38.48 y $39.82{ }^{\circ} \mathrm{C}$. Esto muestra el potencial de calentamiento que tiene este tipo de gramíneas, que se asemeja a materiales como el metal, el concreto y el asfalto. Contrariamente, se observó que a excepción de la imagen 1989/08/07, el café fue la cobertura con la menor TST promedio, indicando una capacidad alta de enfriamiento de la superficie.

Las temperaturas altas encontradas en la cobertura urbana coincide con lo planteado por Weng (2001) sobre el calentamiento de la superficie en las ciudades, que se debe a la sustitución de la vegetación por materiales no evaporantes ni transpirantes como el metal y el concreto, lo que indica que en las zonas con presencia de coberturas arbóreas las temperaturas son menores porque la vegetación densa puede reducir la cantidad de calor almacenado en el suelo y en las estructuras superficiales a través de la evapotranspiración.

Por otra parte, Zhou et al. (2011), aseguran que la temperatura superficial disminuye con la complejidad y la variabilidad de la forma de la vegetación 
leñosa y herbácea; lo que concuerda con los resultados encontrados en este estudio para los pastos, pero difiere de lo encontrado en el café, que corresponde en su gran mayoría con monocultivos a libre exposición solar, a los que a pesar de habérseles disminuido esa complejidad y variabilidad, presentan TST menor a la de la selva Andina, cobertura que desde este punto de vista debería ser la de mayor capacidad de enfriamiento de la superficie.

Estas diferencias entre la temperatura superficial de coberturas urbanas y coberturas vegetales han sido documentadas en varios estudios (CAO et al., 2008; QIAN et al., 2006; KATSIABANI et al., 2009, SOUTHWORTH, 2004), dejando claro que la abundancia de la vegetación es uno de los factores que más influye sobre esta variable, debido a que divide la radiación solar en flujos de calor sensible y calor latente y hace que la interacción entre la dinámica térmica y las plantas creen firmas espectrales únicas en cada tipo de cobertura (WENG et al., 2004).
Los valores promedios de temperatura de superficie coinciden con el estudio realizado por Xiao y Weng (2007) en el que se observó que los valores de TST más altos correspondían con la cobertura urbana. Sin embargo, también encontraron que el bosque presentaba valores altos de TST, incluso superiores a los de las zonas agrícolas y suelo desnudo, lo que difiere de lo observado en la cuenca del río La Vieja, donde la selva Andina se caracterizó por tener valores bajos de TST.

\section{Relación entre IVDN, IADN y TST}

Los índices (IVDN y IADN) y la TST mostraron una correlación negativa (Tabla 3 ), lo que indica que las coberturas con mayores valores en los índices tienen mayor capacidad de enfriamiento de la superficie. El IADN presentó mayor correlación con la temperatura que el IVDN, de manera que el tipo de cobertura y en particular su capacidad de retención de humedad fueron determinantes en las condiciones de la temperatura de superficie. 
Temperatura de la superficie terrestre en diferentes tipos de cobertura de la región andina colombiana

Andrés Felipe Carvajal, José Daniel Pabón

Tabla 2. Medias y desviaciones estándar de IVDN, IADN y TST* por cobertura. Letras diferentes dentro de una misma imagen indican diferencias estadísticamente significativas $(\mathrm{p}<0.01)$ entre las coberturas.

\begin{tabular}{|c|c|c|c|c|c|c|}
\hline Cobertura & IVDN & & IADN & & TST* & \\
\hline & $\mathrm{M}$ & D.E & $\mathrm{M}$ & D.E & $\mathrm{M}$ & D.E \\
\hline \multicolumn{7}{|l|}{$1989 / 08 / 07$} \\
\hline Café & $0.68^{\mathrm{a}}$ & 0.03 & $0.23^{\mathrm{a}}$ & 0.03 & $31.86^{\mathrm{a}}$ & 0.55 \\
\hline Pasto & $0.21 \mathrm{~b}$ & 0.11 & $-0.30 b$ & 0.09 & $38.64 b$ & 2.05 \\
\hline Plantación forestal & $0.64 \mathrm{c}$ & 0.05 & $0.34 \mathrm{c}$ & 0.05 & $33.69 \mathrm{c}$ & 1.92 \\
\hline Selva Andina & $0.57 \mathrm{~d}$ & 0.09 & $0.21 \mathrm{~d}$ & 0.04 & $31.71^{\mathrm{a}}$ & 3.05 \\
\hline Urbana & $-0.03 e$ & 0.08 & $-0.27 \mathrm{e}$ & 0.05 & $38.48 b$ & 0.81 \\
\hline \multicolumn{7}{|l|}{$1997 / 08 / 21$} \\
\hline Café & $0.60^{\mathrm{a}}$ & 0.06 & $0.17^{\mathrm{a}}$ & 0.07 & $33.43^{\mathrm{a}}$ & 0.72 \\
\hline Pasto & $0.19 \mathrm{~b}$ & 0.09 & $-0.31 b$ & 0.09 & $42.27 \mathrm{~b}$ & 1.93 \\
\hline Plantación forestal & $0.58 \mathrm{a}$ & 0.04 & $0.26 \mathrm{c}$ & 0.06 & $37.26 \mathrm{c}$ & 1.01 \\
\hline Selva Andina & $0.57 \mathrm{c}$ & 0.05 & $0.17 \mathrm{~d}$ & 0.05 & $34.95 \mathrm{~d}$ & 2.04 \\
\hline Urbana & $-0.03 \mathrm{~d}$ & 0.07 & $-0.26 \mathrm{e}$ & 0.05 & $39.82 \mathrm{e}$ & 1.34 \\
\hline \multicolumn{7}{|l|}{$1998 / 05 / 20$} \\
\hline Café & $0.64 \mathrm{a}$ & 0.03 & $0.20 \mathrm{a}$ & 0.03 & $33.16^{\mathrm{a}}$ & 0.79 \\
\hline Pasto & $0.33 b$ & 0.12 & $-0.16 b$ & 0.10 & $35.58 \mathrm{~b}$ & 1.78 \\
\hline Plantación forestal & $0.58 \mathrm{c}$ & 0.07 & $0.23 \mathrm{c}$ & 0.07 & $35.87 \mathrm{c}$ & 1.10 \\
\hline Selva Andina & $0.55 \mathrm{~d}$ & 0.09 & $0.18 \mathrm{~d}$ & 0.05 & $35.10 \mathrm{c}$ & 4.36 \\
\hline Urbana & $0.00 \mathrm{e}$ & 0.08 & $-0.25 \mathrm{e}$ & 0.06 & $37.76 \mathrm{~d}$ & 1.65 \\
\hline \multicolumn{7}{|l|}{$2002 / 07 / 26$} \\
\hline Café & $0.52 \mathrm{a}$ & 0.07 & $0.13^{\mathrm{a}}$ & 0.07 & $33.79^{\mathrm{a}}$ & 0.90 \\
\hline Pasto & $0.15 \mathrm{~b}$ & 0.18 & $-0.20 b$ & 0.14 & $37.08 \mathrm{~b}$ & 2.88 \\
\hline Plantación forestal & $0.47 \mathrm{c}$ & 0.09 & $0.21 \mathrm{c}$ & 0.07 & $34.89 \mathrm{c}$ & 0.84 \\
\hline Selva Andina & $0.46 \mathrm{~d}$ & 0.06 & $0.10 \mathrm{~d}$ & 0.05 & $35.44 d$ & 0.81 \\
\hline Urbana & $-0.24 \mathrm{e}$ & 0.08 & $-0.32 \mathrm{e}$ & 0.06 & $39.15 \mathrm{e}$ & 1.24 \\
\hline
\end{tabular}

*Temperatura ajustada para eliminar el efecto de la altitud. M=media. D.E.=desviación estándar.

Organización de los autores.

La alta correlación entre IADN y TST concuerda con lo encontrado por Mallick et al. (2012), quienes identificaron un coeficiente de correlación de Pearson de -0.93 entre estas dos variables. Resultados similares a los de este estudio también fueron obtenidos por Dousset y Gourmelon (2003) para la correlación entre el IVDN y TST, atribuyendo esta correlación alta y negativa a la disponibilidad de humedad por parte de la vegetación, la cual permite que una mayor fracción del flujo neto de radiación sea balanceado por la evapotranspiración y por el flujo de calor latente, disminuyendo de esta manera el flujo de calor sensible y por lo tanto la temperatura de la superficie. 
Tabla 3. Coeficientes de correlación de Pearson entre los índices y la TST

\begin{tabular}{cccc}
\hline Variable & IVDN & IADN & TST \\
\hline IVDN & 1 & & \\
\hline IADN & $0.91 *$ & 1 & \\
\hline TST & $-0.72 *$ & $-0.85^{*}$ & 1 \\
\hline & *Correlación significativa al nivel 0.01. Organización de los autores.
\end{tabular}

En los modelos de regresión se identificó que el IVDN y el IADN explican en un $52 \%$ y un $73 \%$ respectivamente, los cambios en la TST. De acuerdo con las ecuaciones de los modelos de regresión, por cada incremento de 0.1 unidades en el IVDN la temperatura disminuye $1.34^{\circ} \mathrm{C}$, mientras que por cada 0.1 unidades de cambio en el IADN, la temperatura disminuye $1.90^{\circ} \mathrm{C}$ (Figura $4 \mathrm{a}$ y $4 \mathrm{~b}$ ). En cuanto al modelo de regresión entre IADN y IVDN se observó que por cada 0.1 unidades de cambio en el IVDN, el IADN aumenta 0.0759 (Figura 4c), mostrando que coberturas con mayor vigor vegetal tienden a presentar mayor capacidad de retención de humedad en las hojas.

Zhang et al. (2009) en un trabajo realizado para estudiar la isla de calor urbana en una provincia de China, encontraron que la relación entre el IVDN y la temperatura de la superficie era baja y no lineal, argumentando que la temperatura se relacionaba más con el área impermeable de la superficie que con las características del índice de vegetación. Esto se pudo presentar por la realización del trabajo al interior de una ciudad, donde la gran mayoría de la superficie correspondió con cobertura construida y no con cobertura vegetal, contrario a lo encontrado en la cuenca del río La Vieja, donde el análisis a escala de paisaje permitió reconocer la fuerte relación que existe entre los índices de vegetación (IVDN) y de agua (IADN) con la temperatura de la superficie.

\section{Distribución espacial de las coberturas y sus efectos en la temperatura de la superficie}

Los patrones de distribución espacial de las coberturas y la TST fueron similares (Figuras 5 (a), (c); Figura 6 (a), (b)), demostrando que la TST depende directamente del tipo de cobertura y de su respectiva composición.
La heterogeneidad del paisaje en la cuenca dejó ver que las transiciones entre coberturas generan cambios drásticos de TST en una misma zona. Al observar los perfiles de temperatura se notó que la diferencia de TST entre la cobertura de pasto y selva Andina llegó a ser hasta de $9{ }^{\circ} \mathrm{C}$ (Figura 5a). Un patrón similar se identificó en la transición entre pasto y plantación forestal, donde la disminución identificada fue de $8{ }^{\circ} \mathrm{C}$ (Figura 5b).

Se pudo constatar la capacidad alta de enfriamiento que tiene el cultivo de café, encontrando en la transición con la cobertura de pasto, una disminución en la TST hasta de $8^{\circ} \mathrm{C}$ (Figura 5(c)). Esto difiere de los resultados encontrados por Weng et al. (2004), quiénes no reportaron diferencias significativas entre la TST de pastos y coberturas agrícolas. Por otra parte, también fue posible reconocer esta capacidad de enfriamiento en la transición de café a zona urbana, donde las diferencias de TST llegaron a ser hasta de $6{ }^{\circ} \mathrm{C}$ (Figura 6 (a)).

Cuando se contrastó la cobertura urbana con pastos, se encontró un comportamiento diferente al observado en las transiciones anteriores. En este caso, se notó que el pasto presentó TST mayor a la de la cobertura urbana, con un incremento en el perfil hasta de $1.28{ }^{\circ} \mathrm{C}$ (Figura 6(b)). Esto demuestra que la cobertura de pastos tiene mayor capacidad de calentamiento que el área construida, lo que deja ver que las gramíneas a pesar de ser una cobertura vegetal, pueden presentar mayor capacidad de absorción de calor que materiales como el concreto y el hierro. 
Figura 4. Modelos de regresión lineal. a) TST-IVDN. b) TST-IADN. c) IADN-IVDN.
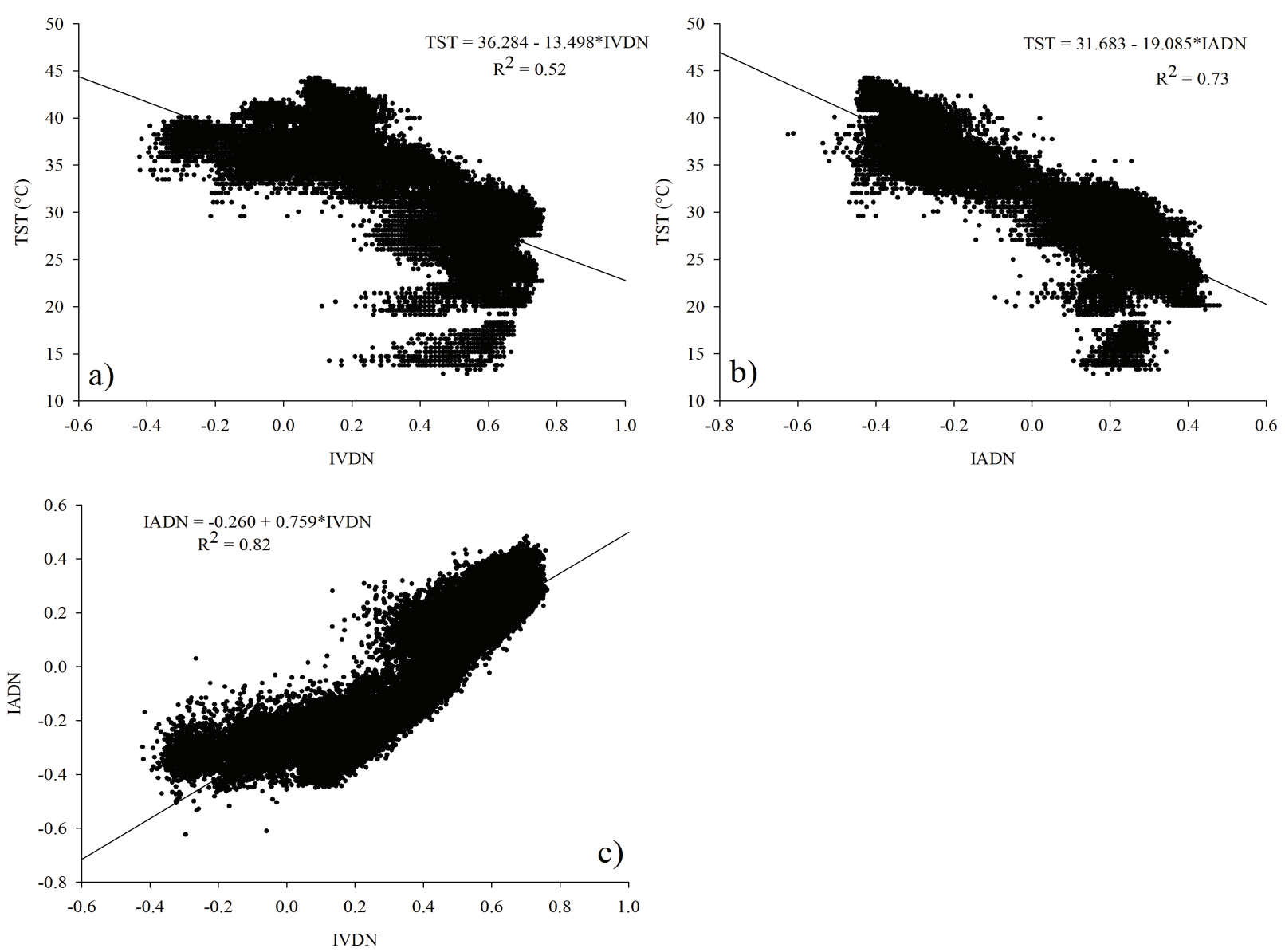

Organización de los autores.

De acuerdo con los hallazgos de este estudio, se confirma lo planteado por Melesse (2004) sobre las bajas temperaturas en la superficie que indican un alto contenido de humedad y/o presencia de cobertura vegetal, y por lo tanto la dominación del calor latente; mientras que las altas temperaturas corresponden con una superficie seca o una vegetación estresada, lo que hace que domine el flujo de calor sensible.

En relación con lo anterior, es importante considerar que no solo se debe hablar de isla de calor urbana, porque según lo encontrado en la cuenca del río La Vieja, existen islas de calor localizadas en las zonas rurales y ocasionadas por la presencia de pastos. Además, se puede decir que dependiendo de la matriz de paisaje que se tenga, se debe hablar de islas de calor o islas de frío. En el caso en que la matriz de paisaje sea de tipo agrícola será posible encontrar islas de calor generadas por zonas urbanas o por pastos, pero si la matriz de paisaje está dominada por pastizales, el análisis se debe realizar de manera inversa, ya que se van a encontrar islas de frío que van a corresponder con la presencia de vegetación arbórea. 
Figura 5. Distribución espacial de las coberturas y la TST, con perfiles de temperatura ilustrando los contrastes de las transiciones entre diferentes tipos de cobertura. a) pasto-selva Andina, b) pasto-plantación forestal c) pasto-café. La línea negra punteada muestra el transecto trazado para elaborar el perfil de temperatura.
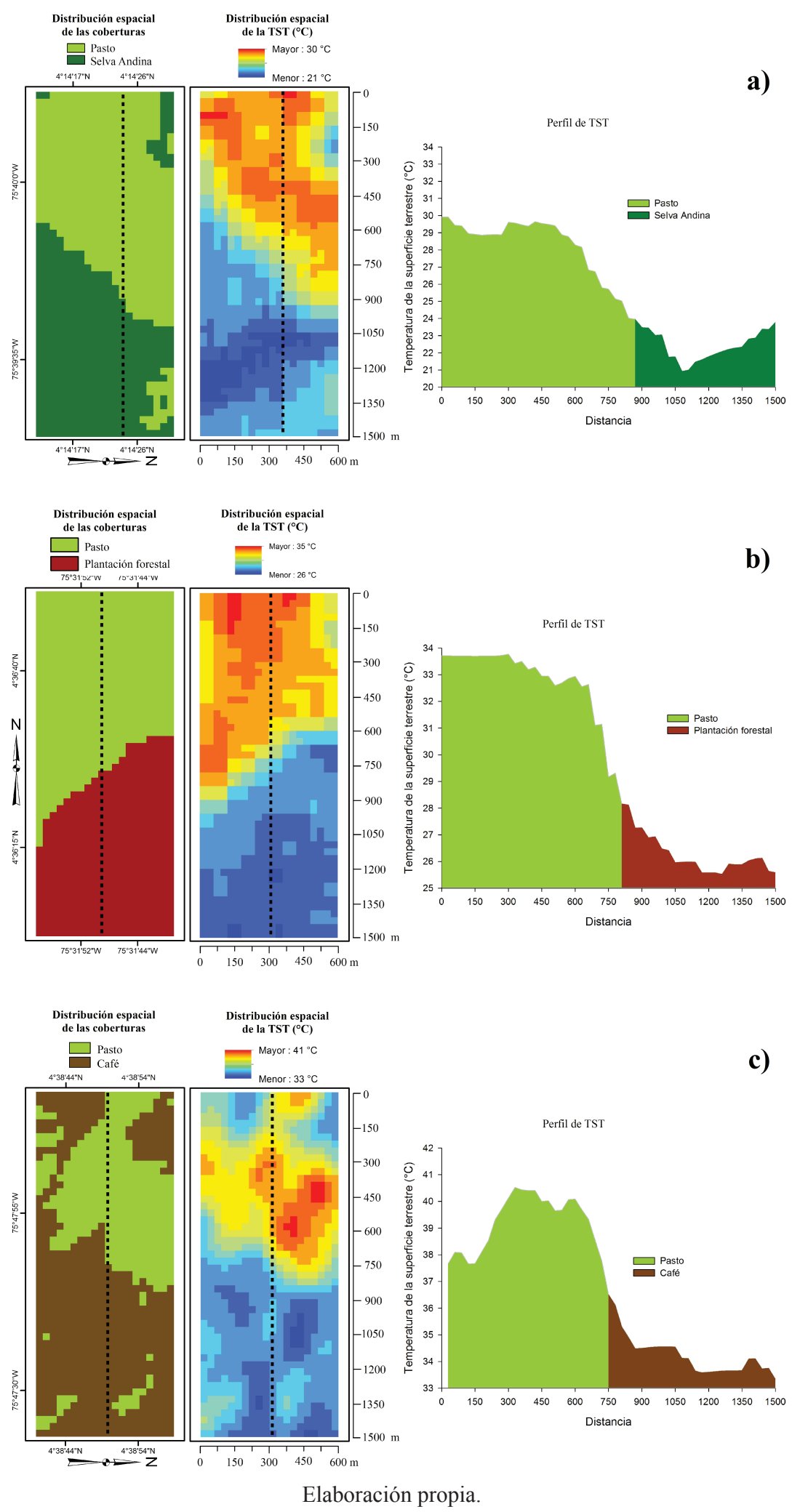

Soc. \& Nat., Uberlândia, 26 (1): 95-112, jan/abr/2014 
Figura 6. Distribución espacial de las coberturas y la TST, con perfiles de temperatura ilustrando los contrastes de las transiciones entre diferentes tipos de cobertura. a) Café- zona urbana, b) Zona urbana-pasto. La línea negra punteada muestra el transecto trazado para elaborar el perfil de temperatura.
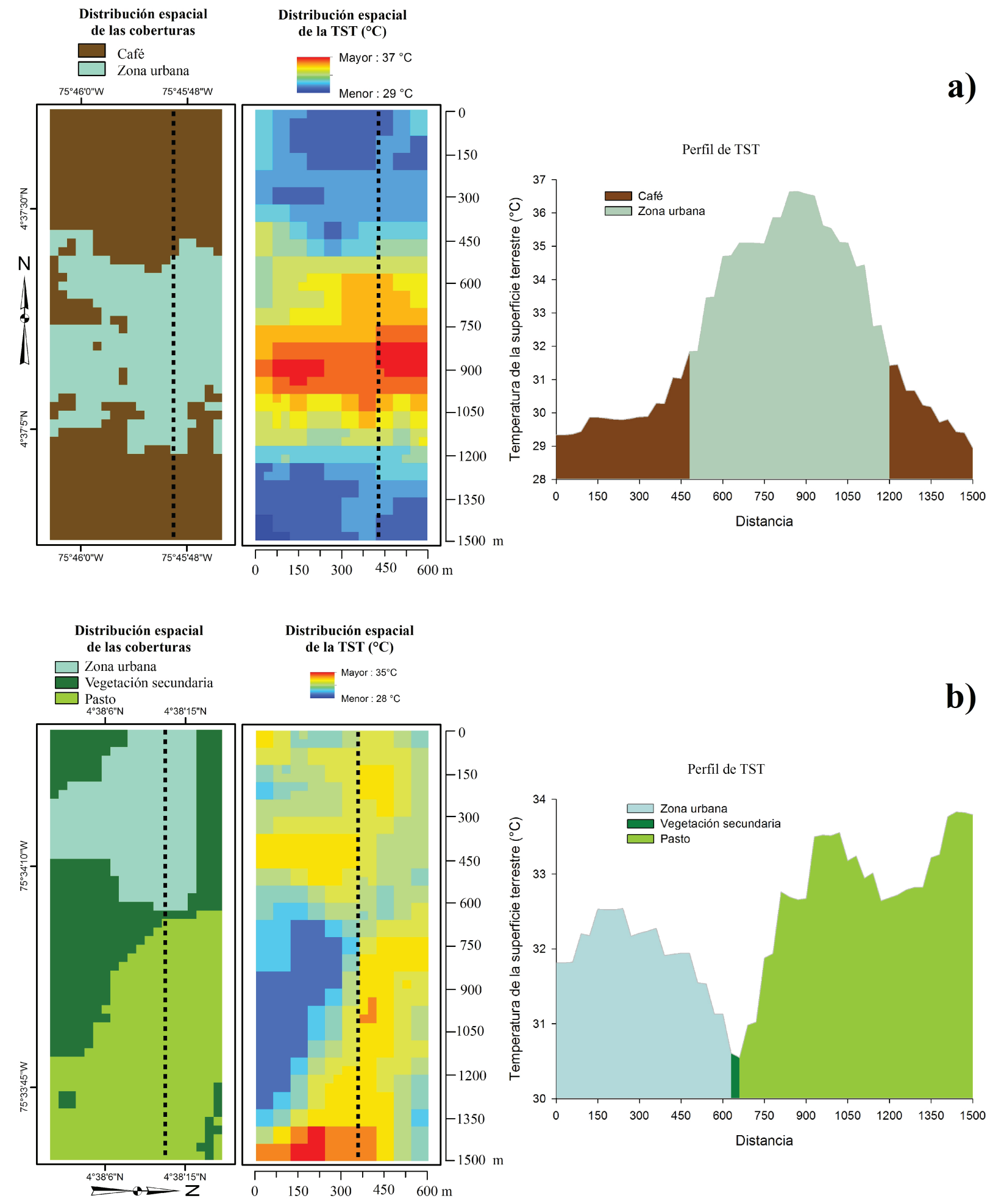

b)

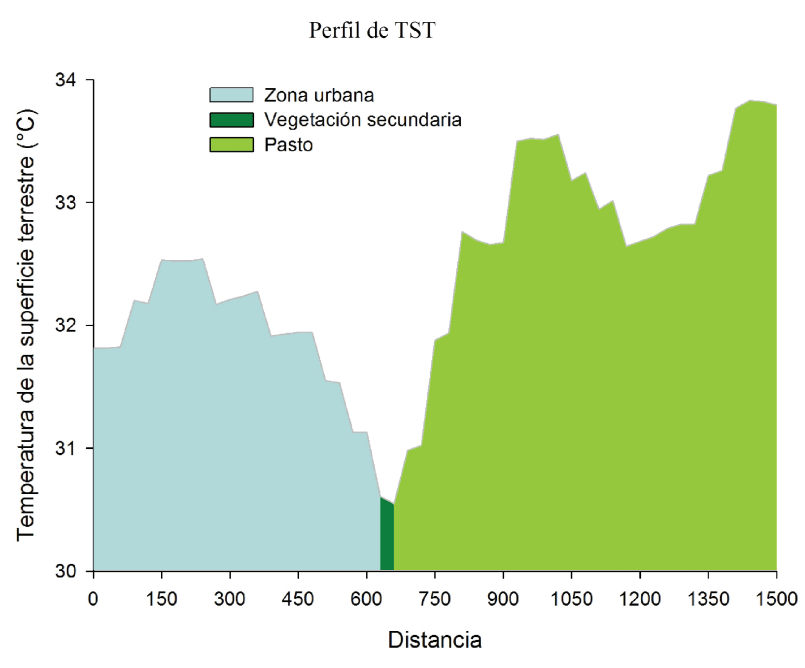

Elaboración propia.

Soc. \& Nat., Uberlândia, 26 (1): 95-112, jan/abr/2014 
Por otra parte, estudios recientes han confirmado que la temperatura superficial está altamente correlacionada con la temperatura del aire, y puede ser utilizada para estimar las temperaturas máximas y mínimas en una región (FU et al., 2011). En esto radica la importancia de una aproximación a la evaluación de la temperatura en superficie de acuerdo con los tipos de cobertura de la tierra en diferentes escalas de análisis, porque permitiría posteriormente identificar tendencias de cambio climático asociadas con procesos de transformación del paisaje. Por lo tanto, se sugiere que en estudios posteriores se correlacionen estas dos variables, con el fin de tener una visión más amplia de los impactos que se pueden generar por la alteración de aspectos como la cobertura fraccional de la vegetación o la sustitución de la misma por materiales artificiales como el concreto y el hierro.

En este orden de ideas, la temperatura superficial e convierte en un indicador de cambio climático de gran importancia, debido a que como plantean Baidya-Roy y Avissar (2002), la heterogeneidad de la superficie terrestre inducida naturalmente o por la acción del ser humano, se representa en forma de parches, cuyas propiedades radiativas y térmicas difieren de las de sus alrededores, lo que puede producir gradientes horizontales de temperatura y presión lo suficientemente fuertes para generar y sostener circulaciones organizadas de mesoescala. Lo anterior, ratifica la importancia de este tipo de estudios para la generación de información relacionada con los impactos de la transformación de las coberturas sobre el clima local y regional, pero no únicamente analizado desde la perspectiva de la isla de calor urbana, porque no solo las superficies completamente transformadas como las ciudades inciden sobre los flujos de calor, también existen coberturas vegetales como los pastos que generan almacenamientos de calor bastante representativos y que pueden afectar las variables climáticas.

Finalmente, se debe enfatizar en que es preocupante encontrar que los pastos poseen una capacidad de calentamiento de la superficie superior al de otras coberturas, porque se estimó que para el año 2000 ya existían a escala global 28 millones de $\mathrm{km}^{2}$ cubiertos por pastizales, correspondientes con el $22 \%$ de la superficie terrestre libre de hielo; mientras que las áreas agrícolas tan solo llegaban a 15 millones de $\mathrm{km}^{2}(12 \%)$
(RAMANKUTTY et al., 2008). Esto es un aspecto de gran interés para la toma de decisiones relacionadas con estrategias de planificación del paisaje, porque la distribución espacial de las coberturas de pasto es más amplia que la de coberturas urbanas, lo que exige seguir haciendo énfasis en las islas de calor de áreas rurales, que se están generando a nivel regional y global como consecuencia de la expansión de los sistemas de producción ganaderos.

\section{CONCLUSIONES}

Del análisis de IVDN y IADN se concluye que son dos índices que permiten diferenciar la cobertura vegetal de la cobertura urbana, y que dentro de las coberturas vegetales facilitan la identificación de aquellas que tienen mayor capacidad de retención de humedad en las hojas. También se concluye que son dos variables importantes en el momento de analizar la relación entre las coberturas y la TST, debido a que se correlacionan de manera significativa y permiten reconocer los tipos de cobertura que por sus características fenológicas favorecen el calentamiento de la superficie terrestre.

Los pastos se caracterizaron por ser la cobertura asociada con las mayores temperaturas de superficie, incluso sobrepasando las temperaturas encontradas en las zonas urbanas. Por el contrario, los cultivos de café mostraron una capacidad alta para el enfriamiento de la superficie, que se asemejó con la selva Andina y las plantaciones forestales. Esto se convierte en un hallazgo importante si se tiene en cuenta que para el año 2002, cerca del 30\% del área de la cuenca se encontraba destinada a actividades agrícolas, principalmente de café, lo que podría estar ayudando con la mitigación del calentamiento superficial generado por los pastos, que para la misma época ya alcanzaban cerca del $35 \%$.

Por lo tanto, se puede afirmar que los sistemas de producción ganadera producen un mayor calentamiento superficial, mientras que coberturas agrícolas como el café tienden a disminuirlo. De esta manera, se demuestra que la relación entre las coberturas y la TST también es una forma de monitorear el calentamiento global asociado con actividades agrícolas y pecuarias, que a través de la transformación de las características de la vegetación pueden llegar a alterar los flujos de calor entre la superficie y la atmósfera, lo que puede 
conllevar a variaciones en el clima local y regional, y posiblemente contribuir con la problemática del calentamiento y el cambio climático global.

Para finalizar, se concluye que en las zonas rurales se están generando islas de calor asociadas con las coberturas de pastos, razón por la cual se hace necesario abordar el tema no solo desde el ámbito urbano. Además, es importante tener en cuenta que las zonas urbanas se encuentran concentradas espacialmente, mientras que la distribución de los pastos es más amplia y puede generar mayores impactos negativos en el balance de radiación tanto a escala regional como global. Desde este punto de vista, es de suma importancia que se tenga en cuenta la configuración del paisaje en la toma de decisiones relacionadas con la mitigación del calentamiento global, porque la expansión de los sistemas ganaderos está generando cambios importantes en las propiedades de la superficie terrestre, que es uno de los componentes fundamentales del sistema climático.

\section{AGRADECIMIENTOS}

Dirección de Investigación sede Bogotá, Universidad Nacional de Colombia sede Bogotá (Proyecto código QUIPU: 201010018165) - Faculdad de Ciencias Humanas, Universidad Nacional de Colombia sede Bogotá (Proyecto código QUIPU: 201010018520) - Departamento Administrativo de Ciencia, Tecnología e innovación - COLCIENCIAS (Beca Francisco José de Caldas año 2009).

\section{REFERENCIAS}

BAIDYA-ROY, S., AVISSAR, R. Impact of land use/ land cover change on regional hydrometeorology in Amazonia. Journal of Geophysical Research, v. 107, n. D20, p. 1-12, 2002.

CAO, L., LI, P., ZHANG, L., CHEN, T. Remote sensing image-based analysis of the relationship between urban heat island and vegetation fraction. The International Archives of the Photogrammetry, Remote Sensing and Spatial Information Sciences, v. 37, p. 1379-1384, 2008.

CHANDER, G., MARKHAM, B. Revised Landsat-5 TM Radiometric Calibration Procedures and Postcalibration Dynamic Ranges. IEEE Transactions on Geoscience and Remote Sensing. v. 41, n. 11, p. 2674-2677, 2003.
CHANDER, G., MARKHAM, B., HELDER, D.L. Summary of current radiometric calibration coefficients for Landsat MSS, TM, ETM+ and EO-1 ALI sensors. Remote Sensing of Environment, v. 113, p. 893-903, 2009.

CHAVEZ, P.S. An Improved Dark-Object Subtraction Technique for Atmospheric Scattering Correction of Multispectral Data. Remote Sensing of Environment, v. 24, p. 459-479, 1988.

COLL, C., CASELLES, V., GALVE, J.M., VALOR, E., NICLÒS, R., SÁNCHEZ, J.M., RIVAS, R. Ground measurements for the validation of land surface temperatures derived from AATSR and MODIS data. $R e-$ mote Sensing of Environment, v. 97, p. 288-300, 2005.

CRQ (Corporación Autónoma Regional del Quindío), et. al.. Plan de ordenamiento y manejo de la cuenca del río La Vieja - Diagnóstico. Quindío: CRQ, 2005, $217 \mathrm{p}$.

DI RIENZO, J.A., CASANOVES, F., BALZARINI, M.G., GONZALEZ L., TABLADA, M., ROBLEDO C.W. InfoStat versión 2012. Grupo InfoStat, FCA, Universidad Nacional de Córdoba, Argentina. Disponible en: $<$ http://www.infostat.com.ar $>$, Acceso en enero de 2012.

DOUSSET, B., GOURMELON, F. Satellite multi-sensor data analysis of urban surface temperaturas and landcover. ISPRS Journal of Photogrammetry \& Remote Sensing, v. 58, p. 43-54, 2003.

ETTER, A., MCALPINE, C., PULLAR, D., POSSINGHAM, H. Modeling the age of tropical moist forest fragments in heavily-cleared lowland landscapes of Colombia. Forest Ecology and Management, v. 208, p. 249-260, 2005.

FU, G., SHEN, Z., ZHANG, X., SHI, P., ZHANG, Y., WU, J. Estimating air temperature of an alpine meadow on the Northern Tibetan Plateau using MODIS land surface temperature. Acta Ecologica Sinica, v. 31, p. 8-13, 2011. 
GAO, B.C. NDWI-A normalized difference water index for remote sensing of vegetation liquid water from space. Remote Sensing of Environment, v. 58, p. 257-266, 1996.

JI, L., PETERS, A.J. Assessing vegetation response to drought in the northern Great Plains using vegetation and drought indices. Remote Sensing of Environment, v.87, p. 85-98, 2003.

JULIEN, Y., SOBRINO, J.A., VERHOEF, W. Changes in land surface temperatures and NDVI values over Europe between 1982 and 1999. Remote Sensing of Environment, v. 103, p. 43-55, 2006.

KATSIABANI, K., ADAKTILOU, N., CARTALIS, C. A generalised methodology for estimating land surface temperatura for non-urban areas of Greece through the combined use of NOAA-AVHRR data and ancillary information. Advances in Space Research, v.43, p. 930-940, 2009.

LI, F., JACKSON, T.J., KUSTAS, W.P., SCHMUGGE, T.J., FRENCH, A.N., COSH, M.H., BINDLISH, C.R.. Deriving land surface temperature from Landsat 5 and 7 during SMEX02/SMACEX. Remote Sensing of Environment, v. 92, p. 521-534, 2004.

MARÍN, A.L., TORO, L.J., URIBE, S.I. Conectividad estructural del paisaje cafetero en la cuenca alta del río San Juan, suroeste antioqueño, Colombia. Boletín de Ciencias de La Tierra, v. 23, p. 43-54, 2008.

MALLICK, J., KANT, Y., BHARATH, B.D. Estimation of land surface temperature over Delhi using Landsat-7 ETM+. The Journal of Indian Geophysical Union, v. 12, n. 3, p. 131-140, 2008.

MALLICK, J., SINGH, C.K., SHASHTRI, S., RAHMAN, A., MUKHERJEE, S. Land surface emissivity retrieval based on moisture index from LANDSAT TM satellite data over heterogeneous surfaces of Delhi city. International Journal of Applied Earth Observation and Geoinformation, v. 19, p. 348-358, 2012.
MARÍN, A.L., TORO, L.J., URIBE, S.I. Conectividad estructural del paisaje cafetero en la cuenca alta del río San Juan, suroeste antioqueño, Colombia. Boletín de Ciencias de la Tierra, v.23, p. 43-54, 2008.

MELESSE, A.M. Spatiotemporal dynamics of land surface parameters in the Red River of the North Basin. Physics and Chemistry of the Earth, v.29, p. 795-810, 2004.

NETELER, M. Estimating daily land surface temperatures in mountainous environments by reconstructed MODIS LST data. Remote Sensing, v. 2, p. 333-351, 2010.

NICHOL, J.E., FUNG, W.Y., LAM, K., WONG, M.S. Urban heat island diagnosis using ASTER satellite images and 'in situ' air temperature. Atmospheric Research, v. 94, p. 276-284, 2009.

OWEN, T. W., CARLSON T. N., GILLIES, R. R. An assessment of satellite remotely-sensed land cover parameters in quantitatively describing the climatic effect of urbanization. International Journal of Remote Sensing, v. 19, n. 9, p. 1663-1681, 1998.

QIAN, L.X., CUI, H.S., CHANG, J. Impacts of land use and cover change on land surface temperature in the Zhujiang Delta. Pedosphere, v. 16, n. 6, p. 681689, 2006.

RAMANKUTTY, N., EVAN, A.T., MONFREDA, C., FOLEY, J.A. Farming the planet: 1. Geographic distribution of global agricultural lands in the year 2000. Global Biogeochemical Cycles, v. 22, p. 1-19, 2008.

ROUSE, J., HAAS, R., SCHELL, J., DEERING, D., \& HARLAN, J. Monitoring the vernal advancement and retrogradation (green wave effect) of natural vegetation. T.I. NASA/GSFC, Final Report (Ed.). Greenbelt, Maryland, 1974. 371 p.

SOBRINO, J.A., LI, Z.L., STOLL, M.P., BECKER, F. Improvements in the split-window technique for land surface temperature determination. IEEE Transactions on Geoscience and Remote Sensing, v. 32, n. 2, p. 243-253, 1994. 
SOUTHWORTH, J. An assessment of Landsat TM band 6 thermal data for analyzing land cover in tropical dry forest regions. International Journal of Remote Sensing, v. 25, p. 689-706, 2004.

SRIVASTAVA, P.K., MAJUMDAR, T.J., BHATTACHARYA, A.K. Surface temperature estimation in Singhbhum Shear Zone of India using Landsat-7 $\mathrm{ETM}+$ thermal infrared data. Advances in Space Research, v. 43, p. 1563-1574, 2009.

STATHOPOULOU, M., CARTALIS, C. Daytime urban heat islands from Landsat ETM + and Corine land cover data: An application to major cities in Greece. Solar Energy, v. 81, p. 358-368, 2007.

STOHLGREN, T.J., CHASE, T.N., PIELKE, R.A., KITTEL, T.G.F., BARON, J.S. Evidence that local land use practices influence regional climate, vegetation and stream flow patterns in adjacent natural areas. Global Change Biology, v. 4, p. 495-504, 1998.

WANG, X., FULLER, D.O., STERNBERG, L.S.L., MIRALLES-WILHELM, F. Foliar nutrient and water content in subtropical tree islands: A new chemohydrodynamic link between satellite vegetation indices and foliar $\delta^{15} \mathrm{~N}$ values. Remote Sensing of Environment, v. 115, p. 923-930, 2011.

WANG, J., RICH, P.M., PRICE, K.P. Temporal responses of NDVI to precipitation and temperature in the central Great Plains, USA. International Journal of Remote Sensing, v. 24, p. 2345-2364, 2003.

WENG, Q. A remote sensing-GIS evaluation of urban expansion and its impact on surface temperature in the Zhujiang Delta, China. International Journal of Remote Sensing, v. 22, n. 10, p. 1999-2014, 2001.

WENG, Q., LU, D., SCHUBRING, J. Estimation of land surface temperature-vegetation abundance relationship for urban heat island studies. Remote Sensing of Environment, v. 89, p. 467-483, 2004.
WILSON, K., PRESSEY, R.L., NEWTON, A., BURGMAN, M., POSSINGHAM, H.,WESTON, C. Measuring and incorporating vulnerability into conservation planning. Environmental Management, v. 35, n. 5 , p. 527-543, 2005.

WILSON, E.H., SADER, S.A. Detection of forest harvest type using multiple dates of Landsat TM imagery. Remote Sensing of Environment, v. 80, p. 385-396, 2002.

XIAN, G., CRANE, M. An analysis of urban thermal characteristics and associated land cover in Tampa Bay and Las Vegas using Landsat satellite data. Remote Sensing of Environment, v. 104, p. 147-156, 2006.

XIAO, H., WENG, Q. The impact of land use and land cover changes on land surface temperature in a karst area of China. Journal of Environmental Management, v. 85, p. 245-257, 2007.

YUAN, F., BAUER, M.E. Comparison of impervious surface area and normalized difference vegetation index as indicators of surface urban heat island effects in Landsat imagery. Remote Sensing of Environment, v.106, p. 375-386, 2007.

ZHANG, Y., ODEH, I.O.A., HAN, C. Bi-temporal characterization of land surface temperature in relation to impervious surface area, IVDN and NDBI, using a sub-pixel image analysis. International Journal of Applied Earth Observation and Geoinformation, v. 11, p. 256-264, 2009.

ZHOU, W., HUANG, G., CADENASSO, M.L. Does spatial configuration matter? Understanding the effects of land cover pattern on land surface temperature in urban landscapes. Landscape and Urban Planning, v. 102, p. 54-63, 2011.

Soc. \& Nat., Uberlândia, 26 (1): 95-112, jan/abr/2014 